\title{
ON THE NORMAL STRUCTURE OF A ONE-POINT STABILIZER IN A DOUBLY TRANSITIVE PERMUTATION GROUP
}

\author{
M. D. ATKINSON and CHERYL E. PRAEGER \\ (Received 8 March 1977) \\ Communicated by M. F. Newman
}

\begin{abstract}
Let $G$ be a doubly transitive permutation group on a finite set $\Omega$, and let $K^{\alpha}$ be a normal subgroup of the stabilizer $G_{\alpha}$ of a point $\alpha$ in $\Omega$. If the action of $G_{\alpha}$ on the set of orbits of $K^{\alpha}$ in $\Omega-\{\alpha\}$ is 2-primitive with kernel $K^{\alpha}$ it is shown that either $G$ is a normal extension of $\operatorname{PSL}(3, q)$ or $K^{\alpha} \cap G_{\gamma}$ is a strongly closed subgroup of $G_{\alpha \gamma}$ in $G_{\alpha}$, where $\gamma \in \Omega-\{\alpha\}$. If in addition the action of $G_{\alpha}$ on the set of orbits of $K^{\alpha}$ is assumed to be 3-transitive, extra information is obtained using permutation theoretic and centralizer ring methods. In the case where $K^{\alpha}$ has three orbits in $\Omega-\{\alpha\}$ strong restrictions are obtained on either the structure of $G$ or the degrees of certain irreducible characters of $G$.
\end{abstract}

Subject classification (Amer. Math. Soc. (MOS) 1970): 20 B 20, 20 B 25.

Consider the following situation: $G$ is a finite permutation group, 2-transitive on a set $\Omega$, for $\alpha \in \Omega$ there is a nontrivial normal subgroup $K^{\alpha}$ of $G_{\alpha}=\left\{g \mid g \in G, \alpha^{g}=a\right\}$, and for $\gamma \neq \alpha$ we denote by $K^{\gamma}$ the unique conjugate of $K^{\alpha}$ contained in $G_{\gamma}=\left\{g \mid g \in G, \gamma^{g}=\gamma\right\}$.

O'Nan $(1975, I)$ observed that in the known groups satisfying these conditions, it seems that for $\gamma \neq \alpha$, either $K^{\alpha} \cap K^{\gamma}=1$ which is equivalent to saying that $K^{\alpha}$ is a T.I. set (that is, $\left(K^{\alpha}\right)^{g} \cap K^{\alpha}$ is $K^{\alpha}$ or 1 for $g$ in $G$ ), or the subgroups $K_{\gamma}^{\alpha}=K^{\alpha} \cap G_{\gamma}$ and $K_{\alpha}^{\gamma}=K^{\gamma} \cap G_{\alpha}$ are equal which is equivalent to saying that $K_{\gamma}^{\alpha}$ is a strongly closed subgroup of $G_{\alpha \gamma}$ in $G_{\alpha}$.

We shall give conditions under which the observation is true; in particular we shall prove the following

THEOREM A. Let $G$ be a 2-transitive permutation group on a set $\Omega$ of $n$ points; for $\alpha$ in $\Omega$ let $K^{\alpha}$ be a nontrivial normal subgroup of $G_{\alpha}$ and let $\Sigma^{\alpha}=\left\{B_{i}^{\alpha} \mid 1 \leqslant i \leqslant t\right\}$ be the set of $K^{\alpha}$-orbits in $\Omega-\{\alpha\}$. Let $K^{\gamma}$ be the unique conjugate of $K^{\alpha}$ in $G_{\gamma}$, for 
$\gamma \neq \alpha$. Assume that $t=\left|\Sigma^{\alpha}\right| \geqslant 3$ and that the action of $G_{\alpha}$ on $\Sigma^{\alpha}$ is 2-primitive with kernel $K^{\alpha}$. Then either

(i) $K_{\gamma}^{\alpha}=K_{\alpha}^{\gamma}$ for $\gamma \neq \alpha$, or

(ii) $P S L(3, t-1) \leqslant G \leqslant P \Gamma L(3, t-1)$, and $\Omega$ is the set of points or lines of $a$ Desarguesian projective plane of order $t-1$, where $t-1$ is a prime power. Also if $\Omega$ is the set of points then $\{\alpha\} \cup B_{1}^{\alpha}$ is a line in the plane. Finally in this case $K^{\alpha} \cap K^{\gamma}=1$ for $\gamma \neq \alpha$.

If we strengthen the conditions on the action of $G_{\alpha}$ on the set of $K^{\alpha}$-orbits in $\Omega-\{\alpha\}$ then we can say more in the case $K_{\gamma}^{\alpha}=K_{\alpha}^{\gamma}$.

THEOREM B. Let $G$ be a 2-transitive permutation group on a set $\Omega$ of $n$ points; for $\alpha$ in $\Omega$ let $K^{\alpha}$ be a non-trivial normal subgroup of $G_{\alpha}$ and let $\Sigma^{\alpha}=\left\{B_{i}^{\alpha} \mid 1 \leqslant i \leqslant t\right\}$ be the set of $K^{\alpha}$-orbits in $\Omega-\{\alpha\}$. Let $K^{\gamma}$ be the unique conjugate of $K^{\alpha}$ in $G_{\gamma}$ for $\gamma \neq \alpha$, and let $\Sigma \gamma=\left\{B_{i}^{\gamma} \mid 1 \leqslant i \leqslant t\right\}$ be the set of $K^{\gamma}$-orbits in $\Omega-\{\gamma)$, where $\alpha \in B_{1}^{\gamma}$.

Assume that $\left|\Sigma^{\alpha}\right|=t \geqslant 3$ and that the action of $G_{\alpha}$ on $\Sigma^{\alpha}$ is 3-transitive with kernel $K^{\alpha}$. If we set $\Sigma=\bigcup_{\alpha \in \Omega} \Sigma^{\alpha}$ then $|\Sigma|=$ th and the action of $G$ on $\Sigma$ is transitive of rank 6 or 7 . Moreover

(i) in the rank 6 case, $K^{\alpha} \cap K^{\gamma}=1$ for $\gamma \neq \alpha$ and $G$ is the group described in Theorem A (ii),

(ii) in the rank 7 case, $K_{\gamma}^{\alpha}=K_{\alpha}^{\gamma}$ for $\gamma \neq \alpha$, and the subdegrees are $1, t-1$, $b, b(t-1), b(t-1), b(t-1) k, b(t-1)(t-1-k)$, for some integer $k$ satisfying $1 \leqslant k \leqslant \frac{1}{2}(t-1)$, where $b=\left|B_{1}^{\alpha}\right|$. Now if $\beta \in B_{1}^{\alpha}$ then $G_{\alpha \beta} / K_{\beta}^{\alpha}$ acts faithfully and 2-transitively on $\Sigma^{\alpha}-\left\{B_{1}^{\alpha}\right\}$ and on $\Sigma^{\beta}-\left\{B_{1}^{\beta}\right\}$ both with degree $t-1$, and the permutation characters for both actions are the same. Also, either these two representations are equivalent and $k=1$, or $G_{\alpha}$ acting on $\Sigma^{\alpha}$ is a 3-transitive group of automorphisms of a symmetric $3-(t, k+1, \lambda)$ design, where either

(a) $t=4 \lambda+4, k=2 \lambda+1$ and the design is a Hadamard 3-design, $\lambda \geqslant 1$, or

(b) $t=(\lambda+2)\left(\lambda^{2}+4 \lambda+2\right)+1=(\lambda+1)\left(\lambda^{2}+5 \lambda+5\right)$, and $k=\lambda^{2}+3 \lambda+1$, $\lambda \geqslant 1$.

REMARKS. In Theorem B (ii) with $k>1$, the symmetric 3-design exists in case (a) if and only if there is a $t \times t$ Hadamard matrix, and it is unknown if the 3-design of case (b) exists for values of $\lambda$ greater than 1 . If $\lambda=1$ in case (b) then the design is a Steiner system on 22 points which admits the Mathieu group $M_{22}$ as a group of automorphisms (see the remarks in Cameron (1973) or Kantor (1974), section 9B). Cameron (1971) (or see Kantor (1974), section 9B) has shown that these designs do not have 3-transitive automorphism groups for certain values of $\lambda$ (for example, if $2<\lambda<103$, or if $\lambda+1$ is a prime power). In case (a) if $\lambda$ is even and the group of automorphisms is 3-transitive, then Norman (1968) has shown that $\lambda=2$. 
We shall prove Theorems A and B in Section 1, and shall continue our discussion of the rank 7 case of Theorem $B$ in Sections 2, 3 and 4. In Section 2 we give the sizes of the intersections of $K^{\alpha}$-orbits and $K^{\beta}$-orbits in terms of two non-negative integer parameters, $c$ and $x$, which are related by a quadratic equation. We shall show that $G$ is a group of collineations of an affine plane of order $t-1$ if the parameter $c=0$; and if $c$ is non-zero then we show that the group induced on a $K^{\alpha}$-orbit cannot be 2-primitive. Next, in Section 3, we study the permutation character $\pi^{\prime}$ of $G$ acting on $\Sigma$. Here, by use of centralizer ring methods, divisibility conditions are obtained which restrict the possibilities for $n$ and the degrees of the constituents of $\pi^{\prime}$. Finally, in Section 4, we consider the special case $t=3$ and prove the following:

THEOREM D. If $G$ is a doubly transitive group of degree $n$ on a set $\Omega$ and $G_{\alpha}$ has a normal subgroup $K$ which has 3 orbits in $\Omega-\{\alpha\}$ which are permuted as $S_{3}$ by $G_{\alpha}$ then one of the following occurs:

(i) $G \cong P S L(3,2)$ of degree 7 ,

(ii) $G$ has a normal subgroup $M, M \cap G_{\alpha}=K$ and $G / M \cong S_{3}$,

(iii) $G$ has nonlinear irreducible characters of degree $x, y, y-1$ such that

(a) $x+y=n+1$,

(b) $2 n(n-1) / x y$ and $x(n-1) /(y-1)$ are each squares of integers,

(c) $n \equiv 4, \bmod 6$.

DefinImions. If $t, v, k, \lambda$ are integers with $t \geqslant 0, k>0, \lambda>0$, and $v>k+1$, then we define a $t-(v, k, \lambda)$ design (or $t$-design) to be a set of $v$ points and a set of blocks with a relation of incidence such that

(i) any block is incident with $k$ points,

(ii) any $t$ distinct points are incident with $\lambda$ blocks.

If we have a $t-(v, k, \lambda)$ design $\mathscr{D}$ then we define the incidence structure $\mathscr{D}_{p}$ as follows: the set of points is the set of points of $\mathscr{D}$ with the point $p$ removed, the set of blocks of $\mathscr{D}_{p}$ is the set of blocks of $\mathscr{D}$ incident with $p$, and incidence is the same as in $\mathscr{D}$. Then $\mathscr{D}_{p}$ is a $(t-1)-(v-1, k-1, \lambda)$ design; $\mathscr{D}$ is called an extension of $\mathscr{D}_{p}$, and $\mathscr{D}_{p}$ is called extendable.

If $t=2$ and if the number of points is the same as the number of blocks we call the design a symmetric 2-design. An extension of a symmetric 2-design is called a symmetric 3-design.

REMINDER. Readers are reminded that if $G$ has a permutation representation on a set $\Omega$ and also on a set $\Omega^{\prime}$, with permutation characters $\Pi$ and $\Pi^{\prime}$ respectively, then

(a) $(1, \Pi)_{G}$ is the number of orbits of $G$ in $\Omega$, where 1 is the principal character of $G$; and 
(b) $\left(\Pi, \Pi^{\prime}\right)_{G}$ is the number of orbits of $G_{\alpha}$ in $\Omega^{\prime}$ where $\alpha \in \Omega$ (and the analogous statements for $\Omega^{\prime}, \Pi^{\prime}$ ).

\section{Proof of Theorems $A$ and $B$}

Let $G, K^{\alpha}, \Sigma^{\alpha}=\left\{B_{i}^{\alpha} \mid 1 \leqslant i \leqslant t\right\}$ et cetera be as defined above, where for $\gamma \neq \alpha$ we assume that $\alpha \in B_{1}^{\gamma}$. We assume that $K^{\alpha}$ is the kernel of the action of $G_{\alpha}$ on $\Sigma^{\alpha}$, and we set

$$
\Sigma=\bigcup_{\alpha \in \Omega} \Sigma^{\alpha}
$$

LEMMA 1.1. (a) If $G_{\alpha}$ is 2-primitive on $\Sigma^{\alpha}$ and if for $\beta \neq \alpha, K_{\beta}^{\alpha} \neq K_{\alpha}^{\beta}$, then $G$ acting on $\Sigma$ is a transitive rank 6 group of degree $n t$ and the orbits of the setwise stabilizer $H^{\alpha}$ of $B_{1}^{\alpha}$ in $\Sigma$ are as shown in the table.

\begin{tabular}{cll}
\hline No. & \multicolumn{1}{c}{ Description } & Size \\
\hline 1 & $B_{1}^{\alpha}$ & 1 \\
2 & $B_{i, 2 \leqslant i \leqslant t}^{\alpha}$ & $t-1$ \\
3 & $B_{1}^{\beta}, \beta \in B_{1}^{\alpha}$ & $b$ \\
4 & $B_{j}^{\beta}, \beta \in B_{1}^{\alpha} 2 \leqslant j \leqslant t$ & $b(t-1)$ \\
5 & $B_{1}^{\gamma}, \gamma \notin\{\alpha\} \cup B_{1}^{\alpha}$ & $b(t-1)$ \\
6 & $B_{j}^{\gamma}, \gamma \notin\{\alpha\} \cup B_{1}^{\alpha}, 2 \leqslant j \leqslant t$ & $b(t-1)^{2}$ \\
\hline
\end{tabular}

(b) If $G_{\alpha}$ is 3-transitive on $\Sigma^{\alpha}$ then $G$ is transitive on $\Sigma$ and the sets $1-5$ above are orbits for $H^{\alpha}$ in $\Sigma$, and the last set is either an orbit or the union of two orbits for $H^{\alpha}$, where $H^{\alpha}$ is the setwise stabilizer of $B_{1}^{\alpha}$ in $G$. Moreover, in the rank 7 case, if $\beta \in B_{1}^{\alpha}$ then $G_{\alpha \beta}$ is 2-transitive on both $\Sigma^{\alpha}-\left\{B_{1}^{\alpha}\right\}$ and $\Sigma^{\beta}-\left\{B_{1}^{\beta}\right\}$, both with degree $t-1$, and with the same permutation character, and the kernel of both representations is $K_{\alpha}^{\beta}=K_{\beta}^{\alpha}$. The 6 th and 7 th orbits have lengths $b(t-1) k$ and $b(t-1)(t-1-k)$ for some integer $k, 1 \leqslant k \leqslant \frac{1}{2}(t-1)$. Either the two representations of $G_{\alpha \beta}$ above are equivalent and then $k=1$, or the action of $G_{\alpha}$ on $\Sigma^{\alpha}$ is a 3-transitive group of automorphisms of a symmetric $3-(t, k+1, \lambda)$ design where either

(i) $t=4 \lambda+4, k=2 \lambda+1$ and the design is a Hadamard 3-design, $\lambda \geqslant 1$; or

(ii) $t=(\lambda+2)\left(\lambda^{2}+4 \lambda+2\right)+1=(\lambda+1)\left(\lambda^{2}+5 \lambda+5\right)$, and $k=\lambda^{2}+3 \lambda+1, \lambda \geqslant 1$.

Proof of Lemma 1.1. Clearly $G$ is transitive on $\Sigma$. Since $G$ is primitive on $\Omega$ it follows that $B_{i}^{\alpha}=B_{i}^{\beta}$ if and only if $\alpha=\beta, i=j$, and so $|\Sigma|=n t$. For any $\gamma$ let $H^{\gamma}$ denote the setwise stabilizer of $B_{1}^{\gamma}$.

First suppose that $G_{\alpha}$ is 2-transitive on $\Sigma^{\alpha}$. Since $H^{\alpha}$ is transitive on $B_{1}^{\alpha}$ and on $\Omega-\left(B_{1}^{\alpha} \cup\{\alpha\}\right)$ clearly sets $1,2,3$ and 4 are orbits for $H^{\alpha}$ in $\Sigma$. Now if $\beta \in B_{1}^{\alpha}$, then $\left(G_{\alpha \beta}\right)^{\Sigma^{\beta}}=G_{\alpha \beta} K^{\beta} / K^{\beta}$, and since $K^{\beta}$ is transitive on $B_{1}^{\beta}$ clearly $G_{\alpha \beta} K^{\beta}=H^{\beta}$ and so $\left(G_{\alpha \beta}\right)^{\Sigma^{\beta}}=\left(H^{\beta}\right)^{\Sigma^{\beta}}$, and similarly $\left(G_{\alpha \beta}\right)^{\Sigma^{\alpha}}=\left(H^{\alpha}\right)^{\Sigma^{\alpha}}$. Thus $G_{\alpha \beta}$ is transitive on 
$\Sigma^{\beta}-\left\{B_{1}^{\beta}\right\}$ and since $H^{\alpha}$ contains $G_{\alpha \beta}$ for $\beta$ in $B_{1}^{\alpha}$, it follows that set 5 is an orbit for $H^{\alpha}$ in $\Sigma$.

Consider the 6th set $X=\left\{B_{j}^{\gamma} \mid 2 \leqslant j \leqslant t, \gamma \notin B_{1}^{\alpha} \cup\{\alpha\}\right\}$. Suppose first that $G_{\alpha}$ is 2-primitive on $\Sigma^{\alpha}$ and that $K_{\gamma}^{\alpha} \neq K_{\alpha}^{\gamma}$ for $\gamma \neq \alpha$. Since $\left(G_{\alpha \gamma}\right)^{\Sigma^{\gamma}}=\left(H^{\gamma}\right)^{\Sigma^{\gamma}}$, it follows that $G_{\alpha \gamma}$ is primitive on $\Sigma^{\gamma}-\left\{B_{1}^{\gamma}\right\}$. Also since $K_{\gamma}^{\alpha} \neq K_{\alpha}^{\gamma}$, then $K_{\alpha}^{\gamma}$ is a normal subgroup of $G_{\alpha \gamma}$ which acts nontrivially on $\Sigma^{\gamma}$, and hence $K_{\gamma}^{\alpha}$ is transitive on $\Sigma^{\gamma}-\{B \gamma\}$. Finally, since $H^{\alpha}$ contains $K_{\gamma}^{\alpha}$ for any $\gamma$ in $\Omega-\left(B_{1}^{\alpha} \cup\{\alpha\}\right)$, it follows that $H^{\alpha}$ is transitive on $X$ and so $G^{\Sigma}$ has rank 6.

Now suppose that $G_{\alpha}$ is 3-transitive on $\Sigma^{\alpha}$. We have shown that sets 1-5 are orbits for $H^{\alpha}$ in $\Sigma$ so we must consider the 6th set $X$. Let $\gamma \in \Omega-\left(B_{1}^{\alpha} \cup\{\alpha\}\right)$, and let $B_{2}^{\alpha}$, say, be the $K^{\alpha}$-orbit containing $\gamma$. Then $G_{\alpha \gamma}$ is 2-transitive on $\Sigma^{\alpha}-\left\{B_{2}^{\alpha}\right\}$ and $H_{\gamma}^{\alpha}$ is the stabilizer of $B_{1}^{\alpha}$ in this action. Also $G_{\alpha \gamma}$ is 2-transitive on $\Sigma^{\gamma}-\left\{B_{1}^{\gamma}\right\}$. For the proof of this lemma only, let $\Pi=1+\chi$, and $\Pi^{\prime}=1+\chi^{\prime}$ be the permutation characters of $G_{\alpha \gamma}$ for the action on $\Sigma^{\alpha}-\left\{B_{2}^{\alpha}\right\}$ and $\Sigma^{\gamma}-\left\{B_{1}^{\gamma}\right\}$ respectively, where 1 is the principal character of $G_{\alpha \gamma}$ and $\chi, \chi^{\prime}$ are irreducible characters of $G_{\alpha \gamma}$ both of degree $t-2$. Then the number of orbits of $H_{\gamma}^{\alpha}$ in $\Sigma \gamma-\left\{B_{1}^{\gamma}\right\}$ is

$$
\left(\Pi, \Pi^{\prime}\right)_{G_{\alpha \gamma}}=1+\left(\chi, \chi^{\prime}\right)_{G_{\alpha \gamma}}
$$

Since $|X|=b(t-1)^{2}$ and since $H^{\alpha}$ is transitive on $\Omega-\left(B_{1}^{\alpha} \cup\{\alpha\}\right)$, it is clear that $H^{\alpha}$ is transitive on $X$ if and only if $H_{\gamma}^{\alpha}$ is transitive on $\Sigma^{\gamma}-\left\{B_{1}^{\gamma}\right\}$ for some and hence any $\gamma \notin B_{1}^{\alpha} \cup\{\alpha\}$. We have shown above that this is true if and only if $\chi \neq \chi^{\prime}$.

Consider the case in which $H^{\alpha}$ is not transitive on $X$. Then $\chi=\chi^{\prime}$ and $H_{\gamma}^{\alpha}$ has two orbits in $\Sigma^{\gamma}-\left\{B_{1}^{\gamma}\right\}$. Let their lengths be $k$ and $t-1-k$ where $1 \leqslant k \leqslant \frac{1}{2}(t-1)$. Then $H^{\alpha}$ has two orbits in $X$ of lengths $b(t-1) k$ and $b(t-1)(t-1-k)$ since $H^{\alpha}$ is transitive on $\Omega-\left(B_{1}^{\alpha} \cup\{\alpha\}\right)$. Since $\Pi=\Pi^{\prime}$ clearly the kernels of the two actions, namely $K_{\gamma}^{\alpha}$ and $K_{\alpha}^{\gamma}$ are the same. Now if $g$ is an element of $G_{\alpha}$ such that $\gamma^{g}=\beta$, then conjugation by $g$ maps $G_{\alpha \gamma}$ to $G_{\alpha \beta}$, and the actions of $G_{\alpha \gamma}$ on $\Sigma^{\alpha}-\left\{B_{2}^{\alpha}\right\}$ and $\Sigma \gamma-\left\{B_{1}^{\gamma}\right\}$ to the actions of $G_{\alpha \beta}$ on $\Sigma^{\alpha}-\left\{B_{1}^{\alpha}\right\}$ and $\Sigma^{\beta}-\left\{B_{1}^{\beta}\right\}$ respectively. So these latter two actions have the same character, the same kernel $K_{\beta}^{\alpha}=K_{\alpha}^{\beta}$, and the setwise stabilizer, say $L$ of $B_{2}^{\alpha}$ in $G_{\alpha \beta}$, has two orbits in $\Sigma^{\beta}-\left\{B_{1}^{\beta}\right\}$ of lengths $k$ and $t-1-k$. Either $k=1$ and the two actions are equivalent, or $k \geqslant 2$ and the translates of the $L$-orbit of length $k$ in $\Sigma^{\beta}-\left\{B_{1}^{\beta}\right\}$ by elements of $G_{\alpha \beta}$ form the set of blocks of a symmetric $2-(t-1, k, \lambda)$ design for $G_{\alpha \beta}$ in $\Sigma^{\beta}-\left\{B_{1}^{\beta}\right\}$. Since $\left(G_{\alpha \beta}\right)^{\Sigma^{\beta}}=\left(H^{\beta}\right)^{\Sigma^{\beta}}$, this design clearly extends to a symmetric 3-design for $G_{\beta}$ in $\Sigma^{\beta}$ with parameters $(t, k+1, \lambda)$ where by Cameron (1973) and Kantor (1974), section 9B, the parameters satisfy either

(i) $t=4 \lambda+4, k=2 \lambda+1$ (a Hadamard 3-design), or

(ii) $t=(\lambda+2)\left(\lambda^{2}+4 \lambda+2\right)+1=(\lambda+1)\left(\lambda^{2}+5 \lambda+5\right)$ and $k=\lambda^{2}+3 \lambda+1$. (The other two conclusions of the theorem of Cameron (1973) do not have 3-transitive automorphism groups as was noted in Kantor (1974), section 9B.) This completes the proof of Lemma 1.1. 
In the remainder of the proof of the theorems we must consider the rank 6 case, and show that the only groups which arise are the 2-dimensional projective linear groups.

Lemma 1.2. Suppose that $\beta \in B_{1}^{\alpha}$, and that $G$ is a transitive rank 6 group on $\Sigma$; suppose either that $G_{\alpha}$ is 2-primitive on $\Sigma^{\alpha}$ with $K_{\beta}^{\alpha} \neq K_{\alpha}^{\beta}$, or that $G_{\alpha}$ is 3-transitive on $\Sigma^{\alpha}$. Then if $d_{i j}=\left|B_{i}^{\alpha} \cap B_{j}^{\beta}\right|$ for $i=1, \ldots, t$ and $j=1, \ldots, t$, the $d_{i j}$ take the following values:

$$
d_{i j}= \begin{cases}b-1-(t-1) c, & \text { if } i=j=1, \\ c, & \text { if } i=1, j>1, \text { or } i>1, j=1 \\ \frac{b-c}{t-1}, & \text { if } i>1, j>1,\end{cases}
$$

where $c$ is a non-negative integer and $c=(b t-t+1 \pm a) / t^{2}$ where

$$
a=\sqrt{ }((t-1)(b t(t-2)+t-1))
$$

is also a non-negative integer.

Proof of Lemma 1.2. Let $H^{\alpha}, H^{\beta}$ be the setwise stabilizers of $B_{1}^{\alpha}, B_{1}^{\beta}$ respectively. Then as we showed in the proof of Lemma 1.1,

$$
\left(G_{\alpha \beta}\right)^{\Sigma^{\alpha}}=\left(H^{\alpha}\right)^{\Sigma^{\alpha}} \text { and }\left(G_{\alpha \beta}\right)^{\Sigma^{\beta}}=\left(H^{\beta}\right)^{\Sigma^{\beta}} .
$$

In particular $G_{\alpha \beta}$ is transitive on $\Sigma^{\alpha}-\left\{B_{1}^{\alpha}\right\}$ and fixes $B_{1}^{\beta}$ so that $d_{i 1}=c$ for some integer $c \geqslant 0$ and any $i \geqslant 2$. Then since $\sum_{i=1}^{t} d_{i 1}=\left|B_{1}^{\beta}-\{\alpha\}\right|=b-1$, if follows that $d_{11}=b-1-(t-1) c$. Similarly since $G_{\alpha \beta}$ fixes $B_{1}^{\alpha}$ and is transitive on $\Sigma^{\beta}-\left\{B_{1}^{\beta}\right\}$ it follows that $d_{1 j}=c$ for $j \geqslant 2$.

Next let $L^{\alpha}$ be the setwise stabilizer in $G_{\alpha}$ of $B_{i}^{\alpha}$ for some fixed $i \geqslant 2$, and let $g$ be an element of $G_{\alpha}$ which interchanges $B_{1}^{\alpha}$ and $B_{i}^{\alpha}$. Then $\beta^{g}=\gamma \in B_{i}^{\alpha},\left(L^{\alpha}\right)^{g}=H^{\alpha}$, and so $\left(L_{\beta}^{\alpha}\right)^{g}=H_{\gamma}^{\alpha}$. In Lemma 1.1 we showed, if either $G_{\alpha}$ is 2-primitive on $\Sigma^{\alpha}$ and $K_{\beta}^{\alpha} \neq K_{\alpha}^{\beta}$, or $G_{\alpha}$ is 3-transitive on $\Sigma^{\alpha}$ and $G$ is rank 6 on $\Sigma$, that $H_{\gamma}^{\alpha}$ is transitive on $\Sigma \gamma-\left\{B_{1}^{\gamma}\right\}$. It follows that $L_{\beta}^{\alpha}$ is transitive on $\Sigma^{\beta}-\left\{B_{1}^{\beta}\right\}$ and so $d_{i j}=x$ for some integer $x \geqslant 0$ and any $j \geqslant 2$. Then since $b=\sum_{i=1}^{t} d_{i j}=c+(t-1) x$, we have $x=(b-c) /(t-1)$. Since this holds for any $i \geqslant 2$, we have found all the $d_{i j}$ in terms of an integer $c \geqslant 0$.

Now by Atkinson (1975), Lemma 2.7

$$
\sum d_{i j}\left(d_{i j}-1\right)=(b-1)(b-2),
$$

where the summation is over all $i=1, \ldots, t$ and $j=1, \ldots, t$. Since $\sum d_{i j}=t b-1$, we have

$$
\Sigma d_{i j}^{2}=b^{2}+(t-3) b+1 \text {. }
$$

On the other hand, by using our calculated values we find that

$$
\sum d_{i j}^{2}=(b-1-(t-1) c)^{2}+2(t-1) c^{2}+(b-c)^{2}
$$


which simplifies to

$$
\sum d_{i j}^{2}=t^{2} c^{2}-2(b t-t+1) c+2 b^{2}-2 b+1 .
$$

Thus,

$$
t^{2} c^{2}-2(b t-t+1) c+b^{2}-(t-1) b=0
$$

and, therefore,

$$
\begin{aligned}
c & =\left[b t-t+1 \pm \sqrt{ }\left\{(b t-t+1)^{2}-t^{2} b(b-t+1)\right\}\right] / t^{2} \\
& =(b t-t+1 \pm a) / t^{2},
\end{aligned}
$$

where $a=\sqrt{ }\{(t-1)(b t(t-2)+t-1)\}$ is a non-negative integer.

Finally, we prove

LEMMA 1.3. Suppose that $G$ is rank 6 on $\Sigma$ and that either $G_{\alpha}$ is 2-primitive on $\Sigma^{\alpha}$ and $K_{\beta}^{\alpha} \neq K_{\alpha}^{\beta}$ for $\beta \neq \alpha$, or $G_{\alpha}$ is 3-transitive on $\Sigma^{\alpha}$. Then

$$
P S L(3, t-1) \leqslant G \leqslant P \Gamma L(3, t-1)
$$

and $\Omega$ is the set of points or lines of a Desarguesian projective plane of order $t-1$, where $t-1$ is a prime power. If $\Omega$ is the set of points then $B_{1}^{\alpha} \cup\{\alpha\}$ is a line, and finally $K^{\alpha} \cap K^{\beta}=1$ for $\alpha \neq \beta$.

Proof of Lemma 1.3. If $G$ is rank 6 on $\Sigma$ then by Lemma 1.1 the subdegrees are $1, t-1, b, b(t-1), b(t-1)$ and $b(t-1)^{2}$. Now let $\Pi=1+\chi$ be the permutation character for the action of $G$ on $\Omega$, where 1 is the principal character and $\chi$ is an irreducible character of degree $t b$. Let $\Pi^{\prime}$ be the permutation character of $G$ on $\Sigma$. Then $\left(\Pi, \Pi^{\prime}\right)$ is the number of orbits of $H^{\alpha}$ in $\Omega$ which is 3 , and since $G$ is transitive on $\Sigma,\left(1, \Pi^{\prime}\right)=1$. Thus $\Pi^{\prime}=1+2 \chi+\varphi$, where $\varphi$ is a character such that $(\Pi, \varphi)=0$. Also since $G$ is rank 6 on $\Sigma, 6=\left(\Pi^{\prime}, \Pi^{\prime}\right)=5+(\varphi, \varphi)$ and so $\varphi$ is an irreducible character and its degree is

$$
n t-1-2(n-1)=(t-2) n+1 .
$$

A remarkable result of Frame (1941) shows that

$$
\frac{(t n)^{4}(t-1)^{5} b^{4}}{(n-1)^{4}((t-2) n+1)}
$$

is an integer. Then since $n=1+t b$ and setting $s=t-1 \geqslant 2$, the above expression becomes

$$
\frac{t^{4}(1+t b)^{4}(t-1)^{5} b^{4}}{(t b)^{4}((t-2)(1+t b)+1)}=\frac{s^{5}(1+(s+1) b)^{4}}{\left(s^{2}-1\right) b+s} .
$$

Now the greatest common divisor of $1+(\mathrm{s}+1) b$ and $\left(s^{2}-1\right) b+s$ is 1 , and so $s^{5}=r\left(s+\left(s^{2}-1\right) b\right)$ for some integer $r \geqslant 1$. Now if $r=1$ then $s^{5}=s+\left(s^{2}-1\right) b$, 
that is,

$$
b=s\left(s^{2}+1\right)=t(t-1)(t-2) .
$$

So assume that $r \geqslant 2$. Then

$$
\begin{aligned}
b & =\left(s^{5} / r-s\right) /\left(s^{2}-1\right) \\
& =s\left(s^{4}-r\right) / r\left(s^{2}-1\right) .
\end{aligned}
$$

Since $\left(s, s^{2}-1\right)=1$, then $s^{2}-1$ divides $s^{4}-r$, that is

and it follows that

$$
s^{2}-1=\left(s^{2}-1, s^{4}-r\right)=\left(s^{2}-1, r-1\right),
$$

$$
r-1=x\left(s^{2}-1\right)
$$

for some positive integer $x$. Thus

If $x=1$ then

$$
\begin{aligned}
b & =s\left(s^{4}-x\left(s^{2}-1\right)-1\right) /\left(x\left(s^{2}-1\right)+1\right)\left(s^{2}-1\right) \\
& =s\left(s^{2}+1-x\right) /\left(x\left(s^{2}-1\right)+1\right) .
\end{aligned}
$$

so assume that $x \geqslant 2$. Now

$$
b=s=t-1
$$

and

$$
\left(s, x\left(s^{2}-1\right)+1\right)=(s, x-1) \leqslant x-1,
$$

$$
\left(s^{2}+1-x, x\left(s^{2}-1\right)+1\right)=\left(s^{2}+1-x, x^{2}-2 x+1\right) \leqslant(x-1)^{2},
$$

and since $b$ is an integer it follows that

$$
x\left(s^{2}-1\right)+1 \leqslant(x-1)^{3} .
$$

Now $b=s\left(s^{2}+1-x\right) /\left(x\left(s^{2}-1\right)+1\right) \leqslant s\left(s^{2}-1\right) /\left(x\left(s^{2}-1\right)+1\right)<s / x$, and substituting in (3) we have

that is,

$$
x\left(s^{2}-1\right)+1<x^{3}<x s^{2} / b^{2} \leqslant x s^{2} / 4,
$$

$$
x\left(\left(3 s^{2} / 4\right)-1\right)+1<0
$$

which yields a contradiction since $s \geqslant 2$. Thus the only possible values for $b$ are given by equations (1) and (2),namely,

or

$$
b=t(t-1)(t-2)
$$

$$
b=t-1 \text {. }
$$

Consider the first case. By Lemma 1.2, as the factors are distinct integers,

$$
\begin{aligned}
a & =\sqrt{ }\left\{(t-1)\left(t^{2}(t-1)(t-2)^{2}+t-1\right)\right\} \\
& =(t-1) \sqrt{ }\left(t^{2}(t-2)^{2}+1\right)
\end{aligned}
$$

is an integer, and hence $t^{2}(t-2)^{2}+1=y^{2}$ for some nonzero integer $y$. Hence $(y-t(t-2))(y+t(t-2))=1$, which is impossible.

In the second case since $d_{11}=(b-1)-(t-1) c \geqslant 0$ and since $b=t-1$ it follows that $c=0$, and so $B_{1}^{\alpha} \cap B_{1}^{\beta}=B_{1}^{\alpha}-\{\beta\}$. Then by Atkinson (1975), Lemma 2.2 it follows 
that $G$ is a group of automorphisms of a 2-design with $\lambda=1$, the blocks of which are the $G$-translates of $B_{1} \cup\{\alpha\}$. Since the number of blocks containing $\alpha$, namely $t$, is the same as the number of points in a block, it follows that the number of blocks is the same as the number of points and the design is symmetric. Then since $\lambda=1$, the design is a projective plane of order $t-1$. It follows from Ostrom and Wagner (1959), Theorem 5, that the plane is Desarguesian and that $P S L(3, t-1) \leqslant G \leqslant P \Gamma L(3, t-1)$ where $t-1$ must be a prime power. Finally, since $B_{i}^{\alpha} \cup\{\alpha\}$ is a line for any $i=1, \ldots, t$, then $K^{\alpha}$ is the kernel of the action of $G_{\alpha}$ on the set of lines containing $\alpha$, and $K^{\alpha}$ is transitive on $B_{i}^{\alpha}$ for each $i=1, \ldots, t$. It can be shown easily that $K^{\alpha} \cap K^{\beta}=1$ for $\beta \neq \alpha$ since distinct lines intersect in exactly one point.

The proofs of both theorems follow from the results of these three lemmas.

\section{Discussion of the rank 7 case of Theorem B}

In this section we assume the hypotheses and notation of Theorem B and we assume that $G$ acts on $\Sigma$ as a transitive rank 7 group. First we obtain a result similar to Lemma 1.2.

Lemma 2.1. Suppose that $\beta \in B_{1}^{\alpha}$ and that $G$ is a transitive rank 7 group on $\Sigma$. If $d_{i j}=\left|B_{i}^{\alpha} \cap B^{\beta}\right|$ for $i=1, \ldots, t$ and $j=1, \ldots, t$, then the $d_{i j}$ take the following values:

$$
d_{i j}= \begin{cases}b-1-(t-1) c & \text { if } i=j=1, \\ c & \text { if } i=1, j>1, \quad \text { or } i>1, j=1, \\ x & k \text { times in the ith row, for any } i>1, \\ \frac{b-c-k x}{t-1-k} & t-1-k \text { times in the ith row for any } i>1,\end{cases}
$$

where $c$ and $x$ are non-negative integers satisfying

$(b-1-(t-1) c)^{2}+2(t-1) c^{2}+(t-1) k x^{2}+\frac{(t-1)(b-c-k x)^{2}}{t-1-k}=b^{2}+(t-3) b+1$, or equivalently

$$
\begin{aligned}
& c^{2}\left(t^{2}-1+\frac{t-1}{t-1-k}\right)-2(t-1) c\left(b-1+\frac{b-k x}{t-1-k}\right) \\
& \left.\quad+(t-1)\left(x^{2} \ldots . t-1-k\right)-2 x k+b^{2}-b(t-1-k)\right) /(t-1-k)=0 .
\end{aligned}
$$

(Actually if $L_{j}^{\beta}$ is the stabilizer of $B_{j}^{\beta}$ in $G_{\alpha \beta}$, then $d_{i j}=x$ if $B_{i}^{\alpha}$ lies in the $L_{j}^{\beta}$-orbit of length $k$ in $\Sigma^{\alpha}-\left\{B_{1}^{\alpha}\right\}$, and $d_{i j}=(b-c-k x) /(t-1-k)$ if $B_{i}^{\alpha}$ lies in the other orbit, where $i>1, j>1$.) 
Proof of Lemma 2.1. Let $H^{\alpha}, H^{\beta}$ be the setwise stabilizers of $B_{1}^{\alpha}, B_{1}^{\beta}$ respectively. Then as in the proof of Lemma 1.2 we obtain the values $d_{11}=b-1-(t-1) c$, $d_{i j}=c$ if $i \neq j$ and $i=1$ or $j=1$, for some integer $c \geqslant 0$.

Let $L^{\alpha}$ be the setwise stabilizer of $B_{i}^{\alpha}$ for some $i \geqslant 2$, and let $g$ be an element of $G_{\alpha}$ which interchanges $B_{1}^{\alpha}$ and $B_{i}^{\alpha}$ so that $\beta^{g}=\gamma \in B_{i}^{\alpha},\left(L^{\alpha}\right)^{g}=H^{\alpha}$ and $\left(L_{\beta}^{\alpha}\right)^{g}=H_{\gamma}^{\alpha}$. By Lemma 1.1, $H_{\gamma}^{\alpha}$ has two orbits in $\Sigma \gamma-\left\{B_{1}^{\gamma}\right\}$ of lengths $k$ and $t-1-k$ respectively (and the possible values of $k$ are given in Lemma 1.1). It follows that $L_{\beta}^{\alpha}$ has two orbits in $\Sigma^{\beta}-\left\{B_{1}^{\beta}\right\}$ of lengths $k, t-1-k$ respectively. Thus $d_{i j}=x$, say, if $B_{j}^{\beta}$ lies in the orbit of length $k$ and $d_{i j}=y$ for $B_{j}^{\beta}$ in the other orbit. Since $\Sigma_{1 \leqslant j \leqslant} d_{i j}=c+k x+(t-1-k) y=b$ we have $y=(b-c-k x) /(t-1-k)$. Clearly $x$ is independent of $i$ (since if $L^{\beta}$ is the stabilizer of $B_{j}^{\beta}$ we find as above that $L_{\alpha}^{\beta}$ has two orbits, of length $k$ and $t-1-k$ respectively, in $\Sigma^{\alpha}-\left\{B_{1}^{\alpha}\right\}$, and $B_{i}^{\alpha}$ lies in the $L_{\alpha}^{\beta}$-orbit of length $k$ if and only if $B_{j}^{\beta}$ lies in the $L_{\beta}^{\alpha}$-orbit of length $k$ ).

Finally, the quadratic equation is obtained by substituting for the $d_{i j}$ in the equation

$$
\sum d_{i j}\left(d_{i j}-1\right)=(b-1)(b-2)
$$

of Atkinson (1975), Lemma 2.7.

LEMMA 2.2. (i) If $K_{\beta}^{\alpha}=K_{\alpha}^{\beta}$ is nontrivial then $b \geqslant t+2$.

(ii) The group $G$ is not a normal extension of the linear group $P S L(m, q)$ in its natural representation for any $m \geqslant 3$ and prime power $q$.

Proof. (i) Since in the rank 7 case $K_{\beta}^{\alpha}=K_{\alpha}^{\beta}$, it follows from O'Nan (1975, II), Theorem $\mathrm{C}$, that $n \leqslant(b-1)^{2}$, and hence $b \geqslant t+2$.

(ii) Suppose that $G$ is a normal extension of $\operatorname{PSL}(m, q)$ for some $m \geqslant 3$. Then $\mathrm{B}_{i}^{\alpha} \cup\{\alpha\}$ is a line containing $\alpha$, and $K^{\alpha}$ is the kernel of the action of $G_{\alpha}$ on the set of lines containing $\alpha$. Hence $K_{\beta}^{\alpha} \neq K_{\alpha}^{\beta}$ for $\alpha \neq \beta$, and $G$ is not rank 7 on $\Sigma$ by Theorem A, which is a contradiction.

THEOREM C. Suppose that $G$ satisfies the hypotheses of Theorem $B$ and that $G$ has rank 7 on $\Sigma$. Then:

(a) The following four conditions are equivalent.

(i) $b<t$.

(ii) The integer $c$ defined in Lemma 2.1 is zero.

(iii) $G$ is an automorphism group of a 2-design with $\lambda=1$, the blocks of which are the translates under $G$ of $B_{1}^{\alpha} \cup\{\alpha\}$.

(iv) $\Omega$ with the translates of $B_{1}^{\alpha} \cup\{\alpha\}$ as lines is an affine translation plane and $G$ contains the group of translations.

(b) If the integer $c$ defined in Lemma 2.1 is nonzero then $b \geqslant t+1, H^{\alpha}$ acts faithfully on $B_{1}^{\alpha}$, and $H^{\alpha}$ is not 2-primitive on $B_{1}^{\alpha}$. Moreover, if $H^{\alpha}$ is 2-transitive on $B_{1}^{\alpha}$ then $c=(b-1) /(t-1) \geqslant 2$ and $\left\{B_{1}^{\alpha} \cap B_{j}^{\beta} \mid 2 \leqslant j \leqslant t\right\}$ is a set of $t-1$ blocks 
of imprimitivity of length $c$ for $G_{\alpha \beta}$ in $B_{1}^{\alpha}-\{\beta\}$, and is permuted 2-transitively by $G_{\alpha \beta}$ with kernel $K_{\alpha}^{\beta}$.

Proof of Theorem C (a). Suppose that $b<t$. Then since the integer $d_{11}$ of Lemma 2.1 is non-negative it follows that $c=0$. Thus $B_{1}^{\alpha} \cap B_{1}^{\beta}=B_{1}^{\alpha}-\{\beta\}$, where $\beta \in B_{1}^{\alpha}$, and so by Atkinson (1975), Lemma 2.2, the $G$-translates of $B_{1}^{\alpha} \cup\{\alpha\}$ form the set of blocks $\mathscr{B}$ of a $2-(n, b+1,1)$ design preserved by $G$. By Fisher's inequality the number $t$ of blocks containing $\alpha$ satisfies $t \geqslant b+1$. (Also $|\mathscr{B}|=n t /(b+1)$ and so $b+1$ divides $t(t-1)$.) Thus it is sufficient to show that the design is an affine plane and (iv) is true. If we can show that the design is an affine plane then the rest of (iv) follows from Ostrom and Wagner (1959), Theorem 1.

By Lemma 2.2(a), $K^{\alpha}$ acts semiregularly on $\Omega-\{\alpha\}$. If $H^{\alpha}$ is not faithful on $B_{1}^{\alpha}$ then the design is an affine plane by Praeger (1978) and Lemma 2.2(b) and so süppose that $H^{\alpha}$ acts faithfully on $B_{1}^{\alpha}$. Let $L$ be the setwise stabilizer of $B=B_{1}^{\alpha} \cup\{\alpha\}$ in $G$. Then $L$ is 2-transitive on $B$ and $L_{\alpha}=H^{\alpha}$ has a normal subgroup $K^{\alpha}$ which acts regularly on $B-\{\alpha\}=B_{1}^{\alpha}$. It follows from Hering, Kantor and Seitz (1972) that $L^{B}$ has a normal subgroup $N$ where either $N$ is regular and elementary abelian, or $L^{B} \leqslant$ Aut $N$ and $N$ is $L_{2}(q), S_{z}(q), U_{3}(q)$ or $R(q)$. If $N$ is one of the simple groups then since $G_{\alpha \beta}=L_{\alpha \beta}$ is faithful on $B$,

$$
\left|G_{\alpha \beta}\right|=\left|L^{B}\right| / b(b+1) \leqslant(b-1) a,
$$

where $q=p^{a}$ for a prime $p$. Now since $a<q$ then

$$
\left|G_{\alpha \beta}\right|<(b-1) q \leqslant(b-1) b \leqslant(t-1)(t-2) .
$$

This is impossible since $G_{\alpha \beta}$ is 2-transitive of degree $t-1$. Thus $N$ is elementary abelian and so $b+1$ is a prime power. Now $\left(N . K^{\alpha}\right)^{B}$ is a sharply 2-transitive Frobenius group with complement $\left(K^{\alpha}\right)^{B}$. It follows that $K^{\alpha}$ has a nontrivial characteristic cyclic subgroup $X$ say, see Gorenstein (1968), 7.6.2, 10.3.1. Then $X$ is normal in $G_{\alpha}$ and is semiregular on $\Omega-\{\alpha\}$. Thus by a result of Aschbacher and and O'Nan (see Kantor (1974), 5B(3)), $G$ has a regular normal subgroup $M$ (for none of the other groups is $G_{\alpha}$ 3-transitive on a set of blocks of imprimitivity).

We shall show now that $G$ acts on $\mathscr{B}$ faithfully as a transitive rank 3 group. Clearly $G$ is transitive on $\mathscr{B}$, and since any point is equal to the intersection of some pair of blocks, it follows that $G$ acts faithfully on $\mathscr{B}$.

Define a map $\varphi: \Sigma \rightarrow \mathscr{B}$ by $\varphi\left(B_{i}^{\gamma}\right)=\{\gamma\} \cup B_{i}^{\gamma}$ for $B_{i}^{\gamma}$ in $\Sigma$. Then $\varphi\left(B_{i}^{\gamma}\right)=\varphi\left(B_{i}^{\beta}\right)$ if and only if either

(i) $\gamma=\beta, i=j$ or

(ii) $\gamma \neq \beta, \gamma \in B_{j}^{\beta}$ (and hence $\beta \in B_{i}^{\gamma}$ ).

Clearly each block in $\mathscr{B}$ is the image of exactly $b+1$ members of $\Sigma$. Now we know that sets 1-5 of Lemma 1.1 are orbits for $H^{\alpha}$ in $\Sigma$, and set 6 is the union of two orbits of $H^{\alpha}$ in $\Sigma$. If $L$ is the setwise stabilizer of $\{\alpha\} \cup B_{1}^{\alpha}$, then $L \geqslant H^{\alpha}$ and $L$ is 
transitive on $\{\alpha\} \cup B_{1}^{\alpha}$. First, the image under $\varphi$ of sets 1 and 3 is the unique block $\{\alpha\} \cup B_{1}^{\alpha}=B_{1}$ say, which is fixed by $L$. Next the image under $\varphi$ of the union of sets 2 and 4 is also an orbit for $L$ since $L$ fixes and is transitive on $B_{1}$. Call it $\mathscr{B}_{2}$. It is easy to see that the $\varphi\left(B_{j}^{\beta}\right)$ are distinct for distinct pairs $(\beta, j)$ where $\beta \in B_{1}$, and $j=2, \ldots, t$, and so $\left|\mathscr{B}_{2}\right|=(b+1)(t-1)$. Now since $\alpha \in B_{1}^{\gamma}$ for $\gamma \neq \alpha$, we have $\{\gamma\} \cup B_{1}^{\gamma}=\{\alpha\} \cup B_{j}^{\alpha}$ where $\gamma \in B_{j}^{\alpha}$ and so $\varphi$ maps set 5 into $\mathscr{B}_{2}$. Also if $\gamma \notin B_{1}$ and if $\beta \in B_{1}^{\alpha}$ then $\gamma \in B_{j}^{\beta}$ for some $j>1$, and $\{\beta\} \cup B_{j}^{\beta}=\{\gamma\} \cup B_{i}^{\gamma}$ where $\beta \in B_{i}^{\gamma}$. Since $\alpha \notin B_{j}^{\beta}$ then $i>1$ and it follows that $\varphi$ maps at least one of the $H^{\alpha}$-orbits in set 6 into $\mathscr{B}_{2}$. If $\varphi$ maps the whole of set 6 into $\mathscr{B}_{2}$ then $\varphi$ maps $(t-1)(1+2 b+b(t-1))$ members of $\Sigma$ into $\mathscr{B}_{2}$. However, since each block is the image of exactly $b+1$ members of $\Sigma$, this number is equal to $(b+1)\left|\mathscr{B}_{2}\right|=(b+1)^{2}(t-1)$. It follows that $t=b+1$. Thus the design is symmetric and so is a projective plane. By Ostrom and Wagner (1959), Theorem 5, it follows that $G$ is a normal extension of $\operatorname{PSL}(3, t-1)$, a contradiction to Lemma 2.2. Hence $G$ acts as a rank 3 group on $\mathscr{B}$. The image under $\varphi$ of one of the $H^{\alpha}$-orbits in set 6 is an $L$-orbit; call it $\mathscr{B}_{3}$.

Suppose that $\varphi$ maps the $H^{\alpha}$-orbit of length $b(t-1) m$ into $\mathscr{B}_{2}$, where $m$ is $k$ or $t-1-k$. Then the number of members of $\Sigma$ mapped into $\mathscr{B}_{2}$, that is $(b+1)^{2}(t-1)$, is equal to $(t-1)(1+2 b+b m)$ and it follows that $b=m$. Since $b+1$ divides $t(t-1)$ and $b \geqslant 2$, and since $m$ is $k$ or $t-1-k$ we obtain the following possibilities:

(i) $b=t-1-k=t-2$ or

(ii) $b=k=\frac{1}{2} t-1$ (Hadamard design) or

(iii) $b=k=\lambda^{2}+3 \lambda+1$, and $t=(\lambda+2)\left(\lambda^{2}+4 \lambda+2\right)+1$, for some positive integer $\lambda$.

In case (i) it follows from Dembowski (1968), p. 21, that the design is an affine plane so assume that (ii) or (iii) is true. Since $|\mathscr{B}|=n t /(b+1)>n$, the normal subgroup $M$ is not transitive on $\mathscr{B}$ and so $G$ acts imprimitively on $\mathscr{B}$. In case (ii), $\left|\mathscr{B}_{2}\right|=\frac{1}{2} t(t-1)>\left|\mathscr{B}_{3}\right|=\left(\frac{1}{2} t-1\right)(t-1)$, so $\left\{B_{1}\right\} \cup \mathscr{B}_{3}$ must be a block of imprimitivity for the action of $G$ on $\mathscr{B}$, and in particular $1+\left|\mathscr{B}_{3}\right|$ divides $\left|\mathscr{B}_{2}\right|$ which is impossible. Similarly in case (iii), $\left|\mathscr{B}_{3}\right|=b(t-1)(t-1-k)>\left|\mathscr{B}_{2}\right|=(b+1)(t-1)$, so $\left\{B_{1}\right\} \cup \mathscr{B}_{2}$ is a block of imprimitivity for $G$ in $\mathscr{B}$ and $1+\left|\mathscr{B}_{2}\right|$ divides $\left|\mathscr{B}_{3}\right|$. A small calculation shows that this is impossible.

Proof of Theorem C(b). If $c \neq 0$ then by part (a), $b \geqslant t$. Suppose that $b=t$. Then since the integer $d_{11}$ of Lemma 2.1 is non-negative and $c>0$ we must have $c=1$. Then by Lemma $2.1, y=(b-c-k x) /(t-1-k)=(t-1-k x) /(t-1-k)$, and $x$ are non-negative integers. An easy calculation shows that

(i) $x=(t-1) / k, y=0$, or

(ii) $x=y=1$, or

(iii) $x=0, \quad y=(t-1) /(t-1-k)$.

The quadratic equation of Lemma 2.1 with $b=t, c=1$ simplifies to

$$
k x^{2}+(t-1-k) y=2 t-3 \text {. }
$$


If $x=y=1$ then $t=2$ whereas we are assuming that $t \geqslant 3$. In cases (i) and (iii) we find that the integers $k$ and $t-1-k$ respectively are equal to $(t-1)^{2} /(2 t-3)$. However, since $(t-1)^{2}$ and $2 t-3$ are coprime it follows that $2 t-3=1$, a contradiction as before. Thus $b \geqslant t+1$. Suppose that $H^{\alpha}$ is 2 -transitive on $B_{1}^{\alpha}$. Then $G_{\alpha \beta}=H_{\beta}^{\alpha}$ is transitive on $B_{1}^{\alpha}-\{\beta\}$. However, since $\left|B_{1}^{\alpha} \cap B_{2}^{\beta}\right|=c \neq 0$ and since $G_{\alpha \beta}$ fixes $B_{1}^{\alpha} \cap B_{1}^{\beta}$ setwise it follows that $\left(B_{1}^{\alpha} \cap B_{1}^{\beta}\right)$ is empty, that is $c=(b-1) /(t-1)>1$. Clearly the set $\left\{B_{1}^{\alpha} \cap B_{j}^{\beta} \mid 2 \leqslant j \leqslant t\right\}$ is a set of $t-1$ blocks of imprimitivity of length $c$ for $G_{\alpha \beta}$ in $B_{1}^{\alpha}-\{\beta\}$, and $G_{\alpha \beta}$ acts 2-transitively on them with kernel $K_{\alpha^{*}}^{\beta}$.

Finally, if $H^{\alpha}$ is not faithful on $B_{1}^{\alpha}$ then by Praeger (1977), Lemma 1.1 and part (a) we have a contradiction. This completes the proof of Theorem $C$.

REMARK. If $H^{\alpha}$ is 2-transitive on $B_{1}^{\alpha}$ we have in addition to the information of Theorem $\mathrm{C}$, the quadratic equation of Lemma 2.1. This, however, seems intractable.

\section{The permutation character of $(G, \Sigma)$}

In this section we continue to assume the hypotheses and notation of the previous section and, in addition, let $\Pi, \Pi^{\prime}$ be the permutation characters for the actions of $G$ on $\Omega, \Sigma$ respectively. Then, as in the proof of Lemma $1.3, \Pi=1+\chi$ and $\Pi^{\prime}=1+2 \chi+\varphi$ where $(\varphi, \Pi)=0$. Here, however, $\varphi$ is not irreducible; since $\left(\Pi^{\prime}, \Pi^{\prime}\right)=7$ we have $(\varphi, \varphi)=2$ and $\varphi=\varphi_{1}+\varphi_{2}$, where $\varphi_{1}$ and $\varphi_{2}$ are irreducible characters of $G$. The degree of $\chi$ is $n-1$ and we shall denote the degrees of $\varphi_{1}, \varphi_{2}$ by $x, y$; so then $x+y=(t-2) n+1$.

We note that the Frame condition demonstrates that

$$
\left\{(n t)^{5} \cdot(t-1) b \cdot b(t-1) \cdot b(t-1) b(t-1) k \cdot b(t-1)(t-1-k)\right\} / x y
$$

is an integer. Using $n-1=b t$ it follows that $\left\{n^{5}(n-1)(t-1)^{5} k(t-1-k)\right\} / x y$ is an integer (which we shall call the Frame parameter). Note that, if $\varphi_{1}$ and $\varphi_{2}$ are rational, the Frame parameter is a perfect square, see Frame (1941).

Lemma 3.1. The degrees $x$ and $y$ are unequal and, in particular, $\varphi_{1}$ and $\varphi_{2}$ are rational.

ProOF. If $x=y=\frac{1}{2}((t-2) n+1)$ the Frame condition yields that

$$
4 b s^{5} k(s-k) /\left(b s^{2}+s-b\right)^{2}
$$

is an integer. In deriving this condition we have used that $n$ and $t$ are coprime with $(t-2) n+1=b t^{2}-2 b t+t-1$, and substituted $s$ in place of $t-1$. We consider the three possibilities for $k$ given by Theorem B. In each case a routine calculation shows that $k(s-k)$ is coprime with $b s^{2}+s-b$. Consequently $4 b s^{5}=r\left(b s^{2}+s-b\right)^{2}$ for some positive integer $r$. We rewrite this as a quadratic in $b$ :

$$
b^{2} r\left(s^{2}-1\right)^{2}+2 b s\left(s^{2} r-r-2 s^{4}\right)+r s^{2}=0 .
$$


The roots of this are

$$
\left(2 s^{5}-s^{3} r+s r \pm 2 s^{3} \sqrt{ } d\right) / r\left(s^{2}-1\right)^{2},
$$

Where $d=s^{4}-r s^{2}+r$, and at least one of the roots is a positive integer. In particular one of $\left(\left(2 s^{5} \pm 2 s^{3} \sqrt{ } d\right) / r\left(s^{2}-1\right)\right)-r s\left(s^{2}-1\right) / r\left(s^{2}-1\right)$ is a positive integer and so one of $\left(2 s^{5} \pm 2 s^{3} \sqrt{ } d\right) /\left(s^{2}-1\right)$ is a positive integer. Since $\left(s, s^{2}-1\right)=1$, one of $\left(2 s^{2} \pm 2 \sqrt{ } d\right) /\left(s^{2}-1\right)$ is a positive integer. If $\left(2 s^{2}-2 \sqrt{ } d\right) /\left(s^{2}-1\right)$ is a positive integer it must be equal to 1 or 2 both of which lead to an easy contradiction. If $\left(2 s^{2}+2 \sqrt{ } d\right) /\left(s^{2}-1\right)$ is a positive integer we have

$$
2<\left(2 s^{2}+2 \sqrt{d}\right) /\left(s^{2}-1\right)<\left(2 s^{2}+2 s^{2}\right) /\left(s^{2}-1\right)=4+4 /\left(s^{2}-1\right) .
$$

Hence $2<\left(2 s^{2}+2 \sqrt{d}\right) /\left(s^{2}-1\right) \leqslant 5$ with equality on the right only if $s=2$. Easy contradictions are obtained in all cases except for $\left(2 s^{2}+2 \sqrt{d}\right) /\left(s^{2}-1\right)=4$ which leads to $b=s=t-1$. In this case we may argue as in the final paragraph of the proof of Lemma 1.3 and obtain a contradiction from Lemma 2.2. This completes the proof of Lemma 3.1.

We now apply to the rank 7 group $(G, \Sigma)$ some of the standard theory of centralizer algebras. An account of this theory may be found in Higman (1972) but we shall briefly discuss the parts which we use. Corresponding to each of the 7 orbits $\Gamma_{i}$ of $G$ on $\Sigma \times \Sigma$ there is an adjacency matrix $A_{i}$ of dimension $t n$. These matrices commute with the permutation matrices $T(g), g \in G$, and span a 7-dimensional algebra $A$.

Suppose that $U$ is some non-singular matrix which reduces the permutation matrices into their irreducible constituents:

$$
U^{-1} T(g) U=\operatorname{diag}(X(g), Y(g), Y(g), Z(g), W(g)),
$$

where $X, Y, Z, W$ are the representations which afford the characters $1, \chi, \varphi_{1}, \varphi_{2}$ respectively. By Schur's lemma the matrices $U^{-1} A_{i} U$ have the form

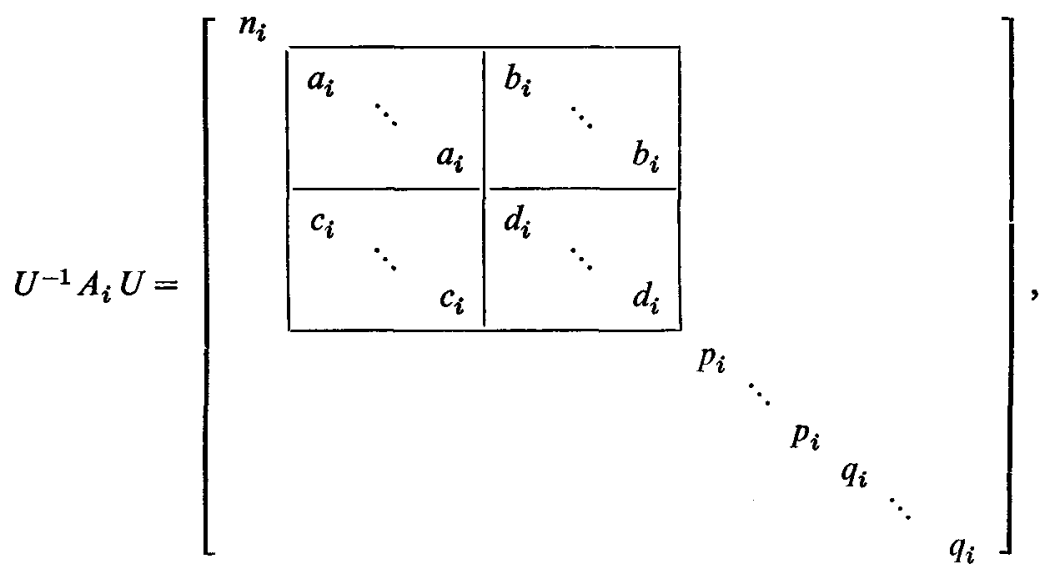


where $a_{i}, b_{i}, c_{i}, d_{i}$ occur $n-1$ times, $p_{i}$ occurs $x$ times and $q_{i}$ occurs $y$ times. It follows that there exists a non-singular matrix $V$ such that

$$
V^{-1} A_{i} V=\operatorname{diag}\left(n_{i}, C_{i}, \ldots, C_{i}, p_{i}, \ldots, p_{i}, q_{i}, \ldots, q_{i}\right),
$$

where $C_{i}=\left(\begin{array}{ll}a_{i} & b_{i} \\ c_{i} & d_{i}\end{array}\right)$ and occurs $n-1$ times.

Following standard practice, we shall use terminology such as "the eigenvalue $p_{i}$ is associated with $\varphi_{1}$ " and "the two eigenvalues of $C_{i}$ are associated with $\chi$ ". As is well known, $n_{i}$, the eigenvalue associated with the character 1 , is the subdegree corresponding to $A_{i}$.

The four representations of the algebra $A$ induced by mapping each $A_{i}$ to $n_{i}$, $C_{i}, p_{i}, q_{i}$ are all irreducible and comprise all the irreducible representations of $A$. The character table of $A$ is a $4 \times 7$ matrix whose rows are indexed by the representations and whose columns are indexed by $A_{1}, A_{2}, \ldots, A_{7}$ and whose $(i, j)$ entry is the trace of the matrix which is the image of $A_{j}$ in the $i$ th representation. It is a consequence of the constituents of $\Pi^{\prime}$ being rational that the entries of this character table are integers. The rows of the character table satisfy certain orthogonality relations. Taking the third row with itself leads to

$$
\frac{p_{1}^{2}}{1}+\frac{p_{2}^{2}}{t-1}+\frac{p_{3}^{2}}{b}+\frac{2 p_{4} p_{5}}{b(t-1)}+\frac{p_{6}^{2}}{b(t-1) k}+\frac{p_{7}^{2}}{b(t-1)(t-1-k)}=\frac{t n}{x}
$$

from which we can deduce that $n(n-1)(t-1) k(t-1-k) / x$ is an integer. A similar calculation with the fourth row produces a corresponding result for $y$ and so we have

LEMMA 3.2. $n(n-1)(t-1) k(t-1-k) / x$ and $n(n-1)(t-1) k(t-1-k) / y$ are integers.

Since $A_{1}$ is the identity matrix all of its $t n$ eigenvalues are equal to 1 . In the next lemma we consider $A_{2}$.

LEMMA 3.3. $A_{2}$ has eigenvalues $t-1$ with multiplicity $n$ and -1 with multiplicity $n(t-1)$. The eigenvalue $t-1$ is associated with the principal character 1 and with $\chi$, and the eigenvalue -1 is associated with $\chi, \varphi_{1}$ and $\varphi_{2}$ except possibly in the following situations:

(i) $t=3$ and one of $x$ and $y$ is equal to 2 ,

(ii) $t=2 b+1$ and one of $x$ and $y$ is equal to $n-1$.

PROOF. The rows and columns of $A_{2}$ are indexed by the points of $\Sigma$ in some order. We shall take an ordering in which, for each $\beta \in \Omega$, the points of $\Sigma^{\beta}$ are adjacent. Then it is clear that $A_{2}$ has the form $\operatorname{diag}(J-I, \ldots, J-I)$ where $I$ and $J$ 
are the $t \times t$ identity and "all ones" matrix, and where $J-I$ occurs $n$ times. Since $J-I$ has eigenvalues $t-1$ with multiplicity 1 and -1 with multiplicity $t-1$ the first part of the lemma follows.

For the second part note first that $t-1$ is associated with the principal character because it is the subdegree. Since $x+y=(t-2) n+1>n$ and the eigenvalue $t-1$ occurs only $n$ times, $t-1$ cannot be associated with both $\varphi_{1}$ and $\varphi_{2}$. We only have to show that it is associated with neither, except possibly in the expectional cases. So suppose, without loss in generality, that the eigenvalue $t-1$ is associate with $\varphi_{1}$. It then follows that $x=n-1$ and $y=(t-3) n+2$.

Then, according to Lemma 3.2,

$$
n b t(t-1) k(t-1-k) /((t-3) n+2) \text { is an integer. }
$$

Since $((t-3) n+2, n)=1$ or 2 and $(t-3) n+2=b t^{2}-3 b t+t-1$ which is coprime with $t$,

$$
2 b(t-1) k(t-1-k) /\left(b t^{2}-3 b t+t-1\right)=m, \text { a positive integer. }
$$

We study this condition in each of the three cases arising from Theorem $B$.

(a) $k=1$. Then $\left({ }^{*}\right)$ becomes $2 b(t-1)(t-2) /\left(b t^{2}-3 b t+t-1\right)=m$ which can be rewritten as $(m-2) b(t-1)(t-2)=m(2 b-t+1)$. If $m>2$ then $m(2 b-t+1)$ is positive and, since it is divisible by $b(t-1)(t-2)$, we have

$$
b(t-1)(t-2)<m(2 b-t+1)<2 m b \text {. }
$$

So $m>\frac{1}{2}(t-1)(t-2)$. But then, from $\left(^{*}\right)$,

$$
2 b(t-1)(t-2)>\frac{1}{2}(t-1)(t-2)\left(b t^{2}-3 b t+t-1\right)
$$

from which it quickly follows that $t<4$ and so $t=3, y=2$ which is one of the exceptional cases. If $m=2$ then $t=2 b+1$ and this is the other exceptional case. The case $m=1$ is easily seen to be impossible.

(b) $k=\frac{1}{2} t-1$. In this case $\left(^{*}\right)$ becomes

$$
2 b(t-1)\left(\frac{1}{2} t-1\right) \frac{1}{2} t /\left(b t^{2}-3 b t+t-1\right)=m
$$

and since $t$ is coprime with the denominator we have

$$
2 b(t-1)(t-2) /\left(b t^{2}-3 b t+t-1\right)=4 m / t
$$

and now an argument as in (a) shows that this is impossible.

$$
\begin{aligned}
k & =\lambda^{2}+3 \lambda+1, \\
t-1-k & =(\lambda+3)(\lambda+1)^{2}, \\
t-1 & =\left(\lambda^{2}+4 \lambda+2\right)(\lambda+2), \\
t-2 & =\left(\lambda^{2}+3 \lambda+1\right)(\lambda+3) .
\end{aligned}
$$

It follows from $\left(^{*}\right)$ that $2 b(t-1)(t-2)(\lambda+1)^{2}=m\left(b t^{2}-3 b t+t-1\right)$ and this may be rewritten as $b(t-1)(t-2)\left(m-2(\lambda+1)^{2}\right)=m(2 b-t+1)$. If $m>2(\lambda+1)^{2}$ then $m(2 b-t+1)$ is positive and, as in $(a), m>\frac{1}{2}(t-1)(t-2)$. But then

$$
2 b(t-1)(t-2)(\lambda+1)^{2}>\frac{1}{2}(t-1)(t-2)\left(b t^{2}-3 b t+t-1\right)
$$


and we can deduce that $4(\lambda+1)^{2}>t^{2}-3 t$. Since $t=(\lambda+1)\left(\lambda^{2}+5 \lambda+5\right)$ this is impossible. If $m<2(\lambda+1)^{2}$ then $b(t-1)(t-2)$ divides the positive number $m(t-2 b-1)$ and so $b(t-1)(t-2)<m(t-1)$. Hence

$$
2(\lambda+1)^{2}>m>b(t-2) \geqslant t-2=\left(\lambda^{2}+3 \lambda+1\right)(\lambda+3)
$$

which is also impossible. If $m=2(\lambda+1)^{2}$ then $t=2 b+1$ which is one of the exceptional cases. This completes the proof of Lemma 3.3.

Before continuing with the general exposition we consider briefly the two exceptional cases in the above lemma. In the first case with $t=3$ we may suppose that $y=2$. Let $M$ be the kernel of $\varphi_{2}$, and let $\mu$ be the 2-dimensional character of $G_{\alpha}$ whose kernel is $K^{\alpha}$ (note that $G_{\alpha} / K^{\alpha} \simeq S_{3}$ ). Then, as

and

$$
\left(\left.\varphi_{2}\right|_{a_{\alpha}}, 1_{a_{\alpha}}+\mu\right)=\left(\left.\varphi_{2}\right|_{a_{\alpha}}, 1_{H^{\alpha}}^{G_{\alpha}^{\alpha}}\right)=\left(\varphi_{2}, 1_{H^{\alpha}}^{G}\right)=\left(\varphi_{2}, \Pi^{\prime}\right)=1
$$

$$
\left(\left.\varphi_{2}\right|_{G_{\alpha}}, 1_{G_{\alpha}}\right)=\left(\varphi_{2}, 1_{G_{\alpha}}^{q}\right)=\left(\varphi_{2}, \Pi\right)=0,
$$

we have $\left.\varphi_{2}\right|_{G_{\alpha}}=\mu$. Thus $M \cap G_{\alpha}=K^{\alpha}$ and, in particular, $G / M \simeq S_{3}$.

In the second case with $t=2 b+1$ we calculate with the Frame condition. The Frame parameter is $n^{5} b(t-1)^{2} k(t-1-k) /(t-2)$. If $k=1$ or if $k=\lambda^{2}+3 \lambda+1$ and $t=(\lambda+1)\left(\lambda^{2}+5 \lambda+5\right)$ then $k(t-1-k) /(t-2)$ is a square. Hence $n b$ is a square. Since $n$ and $b$ are coprime we have $n=i^{2}, b=j^{2}$ and the equation $i^{2}=j^{2}\left(2 j^{2}+1\right)+1$. By a theorem of Siegel, see Mordell (1969), this equation has only a finite number of solutions in integers $i, j$.

In view of the above remarks we shall assume in the remainder of this section that the exceptional conditions of Lemma 3.3 do not hold.

LEMMA 3.4. The adjacency matrices $A_{1}, \ldots, A_{7}$ satisfy the following:

(i) $A_{2} A_{3}=A_{4}$,

(ii) $A_{2} A_{4}=(t-2) A_{4}+(t-1) A_{3}$,

(iii) $A_{2} A_{5}=A_{6}+A_{7}$,

(iv) $A_{2} A_{6}=k A_{5}+(k-1) A_{6}+k A_{7}$,

(v) $A_{2} A_{7}=(t-1-k) A_{5}+(t-1-k) A_{6}+(t-2-k) A_{7}$.

Proof. The only one of these relations which we use subsequently is (i) and so we prove only this; the others are proved in a similar way. Let $B_{i}^{\alpha}$ and $B_{j}^{\beta}$ be a typical $\alpha$-block (that is, an element of $\left.\Sigma^{\alpha}\right)$ and a typical $\beta$-block. The $\left(B_{i}^{\alpha}, B_{j}^{\beta}\right)$ entry of $A_{2} A_{3}$ is the number of $\gamma$-blocks $B_{k}^{\gamma}$ for which $\left(B_{i}^{\alpha}, B_{k}^{\gamma}\right) \in \Gamma_{2}$ and $\left(B_{k}^{\gamma}, B_{j}^{\beta}\right) \in \Gamma_{3}$, where $\Gamma_{i}$ is the $i$ th orbit of $G$ in $\Sigma$ as described in Lemma 1.1. The first condition holds if and only if $B_{l c}^{\gamma}=B_{m}^{\alpha}$ for some $m \neq i$ and so the second condition holds also if and only if $\alpha \in B_{j}^{\beta}$ and $\beta \in B_{m}^{\alpha}$. Hence the $\left(B_{i}^{\alpha}, B_{j}^{\beta}\right)$ entry of $A_{2} A_{3}$ is 0 or 1 and is 1 if and only if $\alpha \in B_{j}^{\beta}$ and $\beta \notin B_{i}^{\alpha}$. This proves (i).

Lemma 3.5. $4 n(n-1)(t-1) b^{2} k(t-1-k) / x y$ is the square of an integer. 
Proof. The character table of the algebra $A$ has the following form:

\begin{tabular}{lcccccccc} 
& & $A_{1}$ & $A_{2}$ & $A_{3}$ & $A_{4}$ & $A_{5}$ & $A_{6}$ & \multicolumn{1}{c}{$A_{7}$} \\
\hline 1 & $\zeta_{1}$ & 1 & $t-1$ & $b$ & $b(t-1)$ & $b(t-1)$ & $b(t-1-k)$ & $b(t-1)(t-1-k)$ \\
$n-1$ & $\zeta_{2}$ & 2 & $t-2$ & & & & & \\
$x$ & $\zeta_{3}$ & 1 & -1 & $p$ & $-p$ & $-p$ & $\frac{1}{2}(p+\alpha)$ & $\frac{1}{2}(p-\alpha)$ \\
$y$ & $\zeta_{4}$ & 1 & -1 & $q$ & $-q$ & $-q$ & $\frac{1}{2}(q+\beta)$ & $\frac{1}{2}(q-\beta)$ \\
\hline
\end{tabular}

The entries in the left-most column are the multiplicities of the correspondin. characters. The entries $\zeta_{i}\left(A_{2}\right)$ follow from Lemma 3.3. Lemma 3.4 shows tha $\zeta_{3}\left(A_{4}\right)=\zeta_{3}\left(A_{2}\right) \zeta_{3}\left(A_{3}\right)$ and the other entries follow from similar consideration together with the relations $\sum_{j} \zeta_{i}\left(A_{j}\right) \approx 0$ if $i>1$, and the relation $A_{4}=A_{5}^{\mathrm{T}}$. We nov use the orthogonality relations on the rows of the character table taking the follow ing pairs: $\left(\zeta_{3}, \zeta_{3}\right),\left(\zeta_{4}, \zeta_{4}\right),\left(\zeta_{3}, \zeta_{4}\right)$. This yields

(i) $b k(t-1-k) t+p^{2}(t+1) k(t-1-k)+\frac{1}{4} p^{2}(t-1)+\frac{1}{4} \alpha^{2}(t-1)+\frac{1}{2} p \alpha(t-1-2 k)$

$$
=n(n-1)(t-1) k(t-1-k) / x,
$$

(ii) $b k(t-1-k) t+q^{2}(t+1) k(t-1-k)+\frac{1}{4} q^{2}(t-1)+\frac{1}{4} \beta^{2}(t-1)+\frac{1}{2} q \beta(t-1-2 k)$

$$
=n(n-1)(t-1) k(t-1-k) / y \text {, }
$$

(iii) $b k(t-1-k) t+p q(t+1) k(t-1-k)+\frac{1}{4} p q(t-1)+\frac{1}{4} \alpha \beta(t-1)$

$$
\begin{aligned}
& +\frac{1}{4}(p \beta+q \alpha)(t-1-2 k) \\
= & 0 .
\end{aligned}
$$

Now, taking (i) $q^{2}+$ (ii) $p^{2}-$ (iii) $2 p q$ gives

(iv) $(n-1) k(t-1-k)(p-q)^{2}+\frac{1}{4}(t-1)(\alpha q-\beta p)^{2}$

$$
=n(n-1)(t-t) k(t-1-k)\left(\frac{q^{2}}{x}+\frac{p^{2}}{y}\right) \text {. }
$$

Next we use the relations satisfied by the columns of the character table. Suppose that $\lambda, \mu$ are the eigenvalues of $C_{3}$ and $\lambda^{\prime}, \mu^{\prime}$ are the eigenvalues of $C_{4}$. Then, as $\operatorname{tr}\left(A_{3}\right)=0$ and $\operatorname{tr}\left(A_{3}^{2}\right)=\operatorname{tnb}$,

$$
\begin{aligned}
b+(n-1)(\lambda+\mu)+x p+y q & =0, \\
b^{2}+(n-1)\left(\lambda^{2}+\mu^{2}\right)+x p^{2}+y q^{2} & =t n b
\end{aligned}
$$

and two similar equations hold for $\lambda^{\prime}, \mu^{\prime}$. Moreover $\operatorname{det} C_{4}=\operatorname{det} C_{2} \cdot \operatorname{det} C_{3}$ and so $\lambda^{\prime} \mu^{\prime}=-(t-1) \lambda \mu$. Eliminating $\lambda, \mu, \lambda^{\prime}, \mu^{\prime}$ between these 5 equations leads to

(v) $n\left(x p^{2}+y q^{2}\right)(t-1)-x y(p-q)^{2}=n b^{2}(t-1)^{2}$.

Combining (iv) and (v) then gives $4 n(n-1)(t-1) b^{2} k(t-1-k) / x y=(\alpha q-\beta p)^{2}$ thereby completing the proof of the lemma.

\section{The case $t=3$}

In this section we consider a doubly transitive group $G$ on a set $\Omega$ where, for $\alpha \in \Omega, G_{\alpha}$ has a normal subgroup $K$ having 3 orbits in $\Omega-\{\alpha\}$. In order to set this in context we begin by noting some related results. 
Lemma 4.1. If $G$ is doubly transitive on $\Omega$ and $G_{\alpha}$ has a normal subgroup with 2 orbits in $\Omega-\{\alpha\}$ then $G$ has a normal subgroup $M$ of index 2 and $M$ is a rank 3 group on $\Omega$ with subdegrees $1, b, b$.

The classification of such groups appears to be a very difficult problem although some progress has been made by Higman (1971).

Lemma 4.2. If $G$ is doubly transitive on $\Omega$ and $G_{\alpha}$ has a normal subgroup $K$ with 3 orbits in $\Omega-\{\alpha\}$ then one of the following occurs:

(i) $G_{\alpha}$ permutes the 3 orbits triply transitively, that is, as $S_{3}$;

(ii) $G$ has a normal subgroup $M$ of index 3 and $M$ is a rank 4 group on $\Omega$ with subdegrees $1, b, b, b$.

Both of these lemmas follow directly from

Lemma 4.3. If $G$ is doubly transitive on $\Omega$ and $G_{\alpha}$ has a normal subgroup $K$ of index $t$ with $t$ orbits in $\Omega-\{\alpha\}$ then $G$ has a normal subgroup $M$ of index $t$ which is a rank $t+1$ group on $\Omega$ with subdegrees $1, b, b, \ldots, b$.

Proof. It is easily seen that $G_{\alpha \beta} \leqslant K$ for any $\beta \neq \alpha$. If $\Pi=1+\chi$ is the permutation character of $G$ then $\Pi=1_{G_{\alpha}}^{G}$ and $\left.\chi\right|_{G_{\alpha}}=1_{G_{\alpha \beta}}^{G_{\alpha}}$.

Let $\theta$ be any non-principal irreducible character of $G_{\alpha}$ with kernel $N_{\theta}$ containing $K$. Then $\left(\theta^{a}, 1_{G}\right)=0$ and $\left(\theta^{\alpha}, \chi\right)=\left(\theta,\left.\chi\right|_{G_{\alpha}}\right)=\left(\theta, 1_{G_{\alpha \beta}}^{G_{\alpha}}\right)=\left(\left.\theta\right|_{G_{\alpha \beta},}, 1_{G_{\alpha \beta}}\right)=\theta(1)$, and so $\theta^{a}=\theta(1) \chi+\xi$ where $\xi$ is some character of degree $\theta(1)$. Since $\theta$ is irreducible, $\left(\theta^{\alpha}, \xi\right)=\left(\theta,\left.\xi\right|_{G_{\alpha}}\right)=1$ and so $\theta=\left.\xi\right|_{G_{\alpha}}$. So if $M_{\theta}=\operatorname{ker} \xi$ then $M_{\theta} \cap G_{\alpha}=N_{\theta}$. Hence if $M=\bigcap_{\theta} M_{\theta}$ (the intersection being taken over all non-principal irreducible characters of $G_{\alpha}$ whose kernel contains $K$ ) then $M \cap G_{\alpha}=K$. As $G$ is doubly transitive $M$ must be transitive and $M_{\alpha}=K$ has orbits of size $1, b, b, \ldots, b$ in $\Omega-\{\alpha\}$.

From now on we assume the hypotheses of Lemma 4.2 and conclusion (i) thus placing ourselves under the hypotheses of the earlier sections. We shall also assume that neither $x$ nor $y$ is equal to 2; this possibility was explored in Section 3 and led to a factor group $S_{3}$ of $G$. Clearly $k=1, b=(n-1) / 3$ and $x+y=n+1$. The conditions on $x, y, n$ derived above are already very restrictive but further conditions can be obtained by considering the subgroup $N$ of index 2 in $G_{\alpha}$ which is the preimage of the Sylow 3-subgroup of $G_{\alpha} / K \simeq S_{3}$ (we change the notation slightly by writing $K$ and $H$ in place of $K^{\alpha}$ and $\left.H^{\alpha}\right)$. Let $\Pi^{\prime \prime}$ be the permutation character for $G$ acting on the set of cosets $\Delta$ of $N$.

Lemma 4.4. $(G, \Delta)$ is a rank 4 group with subdegrees $1,1, n-1, n-1,\left(\Pi, \Pi^{\prime \prime}\right)=2$ and $\left(\Pi^{\prime}, \Pi^{\prime \prime}\right)=4$. 
Proof. Since $N$ is transitive on the set of 3 non-trivial orbits of $K$ in $\Omega, N$ has 2 orbits in $\Omega$. Thus $\left(\Pi, \Pi^{\prime \prime}\right)=2$ and we have $\Pi^{\prime \prime}=1+\chi+\nu$ where $1, \chi$ are not constituents of $\nu$.

Acting on the cosets of $G_{\alpha}, N$ has 2 orbits one of which is $G_{\alpha}$ itself. Each coset of $G_{\alpha}$ contains precisely 2 cosets of $N$. Clearly $N$ has two orbits on the cosets of $N$ contained within $G_{\alpha}$ and therefore $(G, \Delta)$ either has rank 4 with subdegrees $1,1, n-1, n-1$ or has rank 3 . Hence $(v, v)=2$ or 1 , respectively.

Let $\tilde{\Pi}$ be the permutation character for $G$ acting on the cosets of $K$. Then $\tilde{\Pi}=1_{K}^{G}=\left(1_{K}^{G_{\alpha}}\right)^{G}=\left(1_{G_{\alpha}}+\lambda+2 \mu\right)^{G}$ where $1_{G_{\alpha}}, \lambda, \mu$ are the irreducible characters of $G_{\alpha}$ with $K$ in their kernel (corresponding to irreducible characters of $S_{3}$ and of degrees1, 1,2 respectively). Thus

$$
\begin{aligned}
\tilde{\Pi} & =\left(1_{\sigma_{\alpha}}+\lambda\right)^{G}+2 \cdot\left(1_{G_{\alpha}}+\mu\right)^{G}-2.1_{G_{\alpha}}^{G} \\
& =1_{N}^{G}+2.1_{H}^{G}-2.1_{G_{\alpha}}^{G} \\
& =1+3 \chi+\nu+2 \varphi_{1}+2 \varphi_{2} .
\end{aligned}
$$

It follows that $\left(\tilde{\Pi}, \Pi^{\prime}\right)=11+\left(\nu, \varphi_{1}\right)+\left(\nu, \varphi_{2}\right)$. However $\left(\tilde{\Pi}, \Pi^{\prime}\right)$ is the number of orbits of $K$ in $\Sigma$. Three of these orbits are $B_{1}^{\alpha}, B_{2}^{\alpha}, B_{3}^{\alpha}$ and form $\Sigma^{\alpha}$; and the number of orbits of $K$ in $\bigcup_{\beta \in B_{i}^{\alpha}} \Sigma^{\beta}$ is clearly independent of $i$. So ( $\tilde{\Pi}, \Pi^{\prime}$ ) is divisible by 3 . Hence $\left(\tilde{\Pi}, \Pi^{\prime}\right)=12$ and one of $\left(\nu, \varphi_{1}\right)$ and $\left(\nu, \varphi_{2}\right)$ is equal to 1 and the other is zero. Without loss in generality we may assume that $\left(\nu, \varphi_{1}\right)=1$. It follows that $\nu$ is not irreducible because otherwise $\nu=\varphi_{1}, x=n, y=1$. The Frame condition then tells us that $2(n-1)$ is a square and so $n$ is odd. But on the other hand if $L=\operatorname{ker} \varphi_{2}$ then $\left.\Pi^{\prime}\right|_{L}$ contains the principal character twice and so $L$ has two orbits (of equal length) in $\Sigma$ and so $n$ is even.

This proves the lemma and we note that $\nu$ has the form $\varphi_{1}+\varphi_{3}$ where $\varphi_{3}$ is irreducible and of degree $y-1$.

Finally, we show that $\Omega$ can be given the structure of a regular 2-graph (see Seidel (1973) for the relevant background and details). Since $N$ has 2 orbits on cosets of $G_{\alpha}$ and 4 orbits on cosets of $N$, the two cosets of $N$ contained in a single coset of $G_{\alpha}$ are never equivalent under $N$. In particular if $x \in G, n \in N$ and $G_{\alpha} x n=G_{\alpha} x$ then $N x n=N x$, that is, if $n^{x} \in G_{\alpha}$ then $n^{x} \in N$. It follows that Hypothesis A of Hale and Shult (1974) holds. Hale and Shult prove that $G$ acts on a set $\left\{e_{\alpha}\right\}_{\alpha \in \Omega}$ of equiangular lines in the spaces afforded by either $\varphi_{1}$ or $\varphi_{3}$, that is, that $\Omega$ has the structure of a regular 2-graph preserved by $G$. If this 2-graph is trivial then one of $\varphi_{1}$ and $\varphi_{3}$ has dimension 1 . However, we cannot have $x=1$ by the argument given at the end of Lemma 4.4 and we are assuming that $y-1 \neq 1$. So the 2-graph is non-trivial and in particular $n$ is even.

The above discussion enables the proof of Theorem $D$ to be completed easily. Suppose now that $G$ is a group satisfying the hypothesis of Theorem D. Then 
$(G, \Sigma)$ has rank 6 or rank 7 and in the former case Theorem B allows us to conclude that $G \simeq P S L(3,2)$. In the rank 7 case, if the exceptional conditions of Lemma 3.3 hold then $G$ has a normal subgroup $M, M \cap G_{\alpha}=K$ and $G / M \simeq S_{3}$. If the exceptional conditions do not hold then $n$ is even, $n \equiv 1(\bmod 3)$ and so $n \equiv 4(\bmod 6)$. Moreover, as $x+y=n+1$ and, by Lemma 3.2, $x$ and $y$ divide $2 n(n-1), x$ and $y$ are coprime and so $x y$ divides $2 n(n-1)$; by the Frame condition the quotient is a square. We omit the proof that $x(n-1) /(y-1)$ is the square of an integer: it follows by a standard application of centralizer ring theory to $(G, \Delta)$.

We are indebted to Miss Petra Rogers for extensive computer searches for numbers $n, x, y$ satisfying the third conclusion of Theorem $\mathrm{D}$, and for pointing out the following parametric solutions:

(a) $n=\frac{1}{2}\left(p^{2}-1\right)\left(p^{2}-2\right), y=p^{2}-1$, with $p \equiv 3(\bmod 6)$.

(b) $n=(y-1)^{2}$ where $y$ satisfies the "Pellian" condition that $y$ and $2(y-1)$ are each squares.

These are the only solutions which arise for $n \leqslant 10^{8}$.

\section{REFERENCES}

M. D. Atkinson (1975), “Doubly transitive but not doubly primitive permutation groups II", J. London Math. Soc. (2) 10, 53-60.

P. J. Cameron (1971), Structure of suborbits in some primitive permutation groups (Thesis, Oxford University).

P. J. Cameron (1973), “Extending symmetric designs", J. Combinatorial Theory A 14, 215-220.

P. Dembowski (1968), Finite Geometries (Ergebnisse der Math. 44, Springer-Verlag, BerlinHeidelberg-New York).

J. S. Frame (1941), "The double cosets of a finite group", Bull. Amer. Math. Soc. 47, 458-467.

D. Gorenstein (1968), Finite Groups (Harper and Row, New York-Evanston-London).

M. P. Hale Jr. and E. E. Shult (1974), "Equiangular lines, the graph extension theorem and transfer in triply transitive groups", Math. Z. 135, 111-123.

C. Hering, W. M. Kantor and G. M. Seitz (1972), "Finite groups having a split $B N$-pair of rank 1", J. Algebra 20, 435-475.

D. G. Higman (1971), "Solvability of a class of rank 3 permutation groups", Nagoya Math. J. 41, 89-96.

D. G. Higman (1972), Combinatorial Considerations about Permutation Groups (Lecture Notes, Mathematical Institute, Oxford).

W. M. Kantor (1974), "2-Transitive designs", in Combinatorics, Part 3, Combinatorial Group Theory (Proceedings of the Advanced Study Institute on Combinatorics, Breukelen, The Netherlands; ed. M. Hall Jr. and J. H. van Lint, Math. Centre Tracts 57, Amsterdam), pp. 44-97.

L. J. Mordell (1969), Diophantine Equations (Academic Press, London-New York).

C. W. Norman (1968), "A characterization of the Mathieu group $M_{11}$ ", Math. $Z$. 106, 162-166.

M. E. O'Nan (1975), "Normal structure of the one-point stabilizer of a doubly-transitive permutation group I", Trans. Amer. Math. Soc. 214, 1-42; II, ibid., 43-74.

I. G. Ostrom and A. Wagner (1959), "On projective and affine planes with transitive collineation groups”, Math. Z. 71, 186-199.

C. E. Praeger (1977), "Doubly transitive permutation groups which are not doubly primitive", J. Algebra 44, 389-395. 
C. E. Praeger (1978), "Doubly transitive permutation groups in which the one point stabiliser is triply transitive on a set of blocks", J. Algebra (to appear).

J. J. Seidel (1973), “A survey of two-graphs", Proc. Int. Coll. Theorie Combinatorie Acc. Naz. Lincei (Rome).

H. Wielandt (1964), Finite Permutation Groups (Academic Press, London-New York).

Department of Computing Mathematics

University College

Cardiff, Wales

Department of Mathematics

University of Western Australia

Nedlands, Australia 6009 